\title{
含偶氮苯侧链两亲性交替共聚物的合成及自组装
}

\author{
吴嘉诚刘争卉姚远林绍梁* \\ (华东理工大学材料科学与工程学院 上海市先进聚合物材料重点实验室 上海 200237)
}

\begin{abstract}
摘要 通过叠氮一炔点击化学反应, 合成了一种新型的偶氮苯侧链两亲交替共聚物聚(三缩四乙二醇-偶氮苯二乙胺)

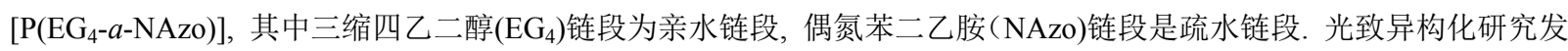
现, 由于特殊的交替拓扑结构, $\mathrm{P}\left(\mathrm{EG}_{4}-a-\mathrm{NAzo}\right)$ )胶束状态不产生有序的偶氮苯堆叠. $\mathrm{P}\left(\mathrm{EG}_{4}-a-\mathrm{NAzo}\right)$ 可以在低浓度溶液中 组装为蠕虫状聚集体. 这种新型的偶氮苯共聚物为光功能性聚合物的分子设计提供了新的思路.
\end{abstract}

关键词 点击反应; 交替共聚物; 偶氮苯; 自组装

\section{Synthesis and Self-Assembly of Alternating Amphiphilic Copolymer with Azobenzene Pendants}

\author{
$\mathrm{Wu}$, Jiacheng Liu, Zhenghui Yao, Yuan Lin, Shaoliang* \\ (Shanghai Key Laboratory of Advanced Polymeric Materials, School of Materials Science and Engineering, \\ East China University of Science and Technology, Shanghai 200237)
}

\begin{abstract}
A novel alternating amphiphilic copolymer poly(tetra glycol- $a$ - $N, N$-bis[2-(1H-1,2,3-triazol-1-yl)ethyl]-4-phenyl-

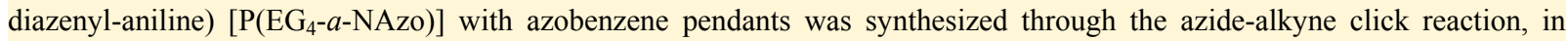
which the hydrophilic unit was tetra glycol $\left(\mathrm{EG}_{4}\right)$ and $N, N$-bis[2-(1H-1,2,3-triazol-1-yl)ethyl]-4-phenyldiazenyl-aniline (NAzo) performed the hydrophobic unit. $\mathrm{P}\left(\mathrm{EG}_{4}-a-\mathrm{NAzo}\right)$ could self-assemble into worm-like aggregate in aqueous solution with initially low concentration. Because of its unique alternating topologies, the azobenzene moiety of $\mathrm{P}\left(\mathrm{EG}_{4}-a\right.$-NAzo $)$ micelle could not pile up orderly. This novel azobenzene copolymer has arisen new thoughts and approaches for the molecular design of photo-functional polymers.

Keywords click reaction; alternating copolymer; azobenzene; self-assembly
\end{abstract}

由于可以简单高效地构筑稳定的微纳米级别结构, 两亲性嵌段共聚物(BCP)的自组装多年来一直受到科研 工作者的广泛关注 ${ }^{[1]}$. 通过调节两亲性嵌段共聚物中亲 水链段与疏水链段的嵌段比, 可以得到一系列丰富多样 的聚集体形貌结构 ${ }^{[2]}$. 同时, 将一系列功能性基团, 例 如偶氮苯、胺基、統基等引入到嵌段共聚物中, 可以使 其组装体拥有对光、 $\mathrm{pH}$ 、温度以及氧化还原等刺激的响 应性 ${ }^{[3]}$. 目前, 功能性嵌段共聚物已在苂光标记 ${ }^{[4]}$ 、智能 载体 ${ }^{[5]}$ 、分子马达 ${ }^{[6]}$ 、微图案阵列 ${ }^{[7]}$ 等领域有了广泛应用. 但是, 嵌段聚合物各段的分子量及分布难以精确控制, 因此很难得到形貌唯一, 尺寸均匀的组装体.

近年来, 科研工作者们制备了一系列类似嵌段共聚 物的聚合物, 即线型两亲性交替共聚物(AAC). 这种聚
合物由两种分子量确定, 亲疏水性不同的结构单元 $\mathrm{A}$ 和 $\mathrm{B}$ 交替连接而成，相较于普通嵌段共聚物， $\mathrm{AAC}$ 特殊的 拓扑结构赋予其链段严格的亲疏水比 ${ }^{[8]}$, 从而使其具备 独特的自组装行为, 可以对组装体的结构进行精确调 控. 如 Zhou 课题组 ${ }^{[9]}$ 通过颈基-环氧的点击(click)反应, 使用双官能团单体 1,4-丁二硫醇和双环氧化丁二烯合成 了 $\mathrm{P}(\mathrm{DHB}-a-\mathrm{BDT})$. 它可自组装形成超细纳米管, 再与 小分子偶联, 可使纳米管带有不同种类的电荷. 该课题 组 ${ }^{[10]}$ 还使用两种反应性单体, 通过乳液聚合诱导自组 装(EAPISA) 获得了微米尺度的多级结构海胆状聚集体. 但是，目前两亲交替拓扑结构的刺激响应型聚合物还鲜 有报道，这也限制了 $\mathrm{AAC}$ 的进一步应用.

本工作采用叠氮-炔点击化学反应 ${ }^{[1]}$, 制备了一种

\footnotetext{
* Corresponding author. E-mail: slin@ecust.edu.cn

Received May 10, 2019; revised May 26, 2019; published online May 28, 2019.

Project supported by the National Natural Science Foundation of China (Nos. 51622301, 51573088, 51873061, 91834301).

国家自然科学基金(Nos. 51622301, 51573088, 51873061, 91834301)资助项目.
} 
新型的含偶氮苯侧链的两亲性交替共聚物聚(三缩四乙 二醇-偶氮苯二乙胺) $\left[\mathrm{P}\left(\mathrm{EG}_{4}-a-\mathrm{NAzo}\right)\right]$, 其中 $\mathrm{EG}_{4}$ 链段为 亲水链段, $\mathrm{NAzo}$ 链段是疏水链段. $\mathrm{P}\left(\mathrm{EG}_{4}-a-\mathrm{NAzo}\right)$ 可以 在低浓度溶液中组装为蠕虫状聚集体. 光致异构化研究 发现, $\mathrm{P}\left(\mathrm{EG}_{4}-a-\mathrm{NAzo}\right)$ 具有特殊的氨基型偶氮苯结构, 由 于叔胺基的给电子效应, 反式偶氮苯结构的吸收峰发生 较大红移至 $405 \mathrm{~nm}$ 处, 且 $\pi-\pi$ 共轭与 $n-\pi$ 共轭部分重叠, 其顺式结构的吸收峰被掩盖. 同时, $\mathrm{P}\left(\mathrm{EG}_{4}-a-\mathrm{NAzo}\right)$ 特殊 的交替拓扑结构使得偶氮苯基团难以有序聚集, 因此 $\mathrm{P}\left(\mathrm{EG}_{4}-a-\mathrm{NAzo}\right)$ 的胶束水溶液中的紫外最大吸收峰与其 $N, N$ 二 二甲基甲酰胺(DMF) 溶液的吸收峰相比, 几乎未发 生红移或蓝移. $\mathrm{P}\left(\mathrm{EG}_{4}-a-\mathrm{NAzo}\right)$ 的成功合成丰富了基于 偶氮苯单元的聚合物的结构与类型, 不仅为功能型交替 共聚物的研究和制备提供了新的策略, 也为拓展偶氮苯 共聚物的应用提供新的思路.

\section{1 结果与讨论}

\section{1 含偶氮苯侧链两亲性交替共聚物 $P\left(E G_{4}-a-N A z o\right)$ 的合成及表征}

两亲性交替共聚物 $\mathrm{P}\left(\mathrm{EG}_{4}-a-\mathrm{NAzo}\right)$ 的合成路线如 Scheme 1 所示. 其中疏水单元单体的合成采用 $N, N$ 二 羟乙基苯胺 $(N$-phenyl diethanolamine)作为反应底物, 通 过对甲苯磺酰氯( $\mathrm{TsCl}$ )的取代引入叠氮基团, 然后重氮 化反应生成偶氮苯二叠氮乙胺 $\left(\mathrm{NAzo}-\mathrm{N}_{3}\right)$; 亲水单元单 体的合成采用三缩四乙二醇 $\left(\mathrm{EG}_{4}\right)$ 作为原料, 通过溴丙
炔取代羟基引入炔基基团，得到端丙炔基三缩四乙二醇 $\left(\mathrm{PAEG}_{4}\right)$. 最后通过叠氮一炔 click 反应制备含偶氮苯侧 链两亲性交替共聚物 $\mathrm{P}\left(\mathrm{EG}_{4}-a-\mathrm{NAzo}\right)$.

NAzo-N ${ }_{3}, \mathrm{PAEG}_{4}$ 以及 $\mathrm{P}\left(\mathrm{EG}_{4}-a-\mathrm{Azo}\right)$ 的核磁共振氢 谱 $\left({ }^{1} \mathrm{H}\right.$ NMR)如图 1a 1c 所示. 图 1c 中 $\delta 7.45$ 处的三氮 唑环峰出现证明了 Click 反应成功进行. 通过凝胶渗透 色谱(GPC)对所得的 $\mathrm{P}\left(\mathrm{EG}_{4}-a-\mathrm{NAzo}\right)$ 进行了分子量和分 子量分布的表征, 图 $1 \mathrm{~d}$ 为 $\mathrm{P}\left(\mathrm{EG}_{4}-a-\mathrm{NAzo}\right)$ 的保留时间曲 线. GPC 结果表明, 聚合物分子量为 $M_{\mathrm{n}}=1.41 \times 10^{4}$ $\mathrm{g} \cdot \mathrm{mol}^{-1}$, 分子量分布(PDI)为 1.24. 根据 GPC 结果, 计 算得平均聚合度 $n=22$. 由于合成为逐步聚合, 反应即 使进行较为完全, 也会有一部分低分子量的产物存在, 因此会产生图 1d 中的小峰.

\section{2 交替共聚物 $P\left(E G_{4}-a-N A z o\right)$ 的光致异构化行为}

通过紫外-可见(UV-Vis)吸收光谱研究了 $\mathrm{P}\left(\mathrm{EG}_{4}-a\right.$ $\mathrm{NAzo}$ )溶液及其胶束水溶液的光致异构化行为. 图 $2 \mathrm{a}$ 为 $\mathrm{P}\left(\mathrm{EG}_{4}-a-\mathrm{NAzo}\right) \mathrm{DMF}$ 溶液被波长 $450 \mathrm{~nm}$ 的蓝光照射不 同时间后的紫外-可见吸收光谱. 对应偶氮苯生色团中 电子的 $\pi \rightarrow \pi *$ 跃迁的反式结构吸收峰位于 $405 \mathrm{~nm}$ 处, 这 是由于叔胺基的给电子效应使得反式偶氮苯结构的吸 收峰发生较大红移 ${ }^{[13]}$, 与顺式结构的 $\mathrm{n} \rightarrow \pi^{*}$ 跃迁吸收峰 部分重叠 ${ }^{[14]}$. 当使用波长 $450 \mathrm{~nm}$ 的蓝光照射时, $405 \mathrm{~nm}$ 处反式偶氮苯的吸收峰随光照时间的延长逐渐减弱, 连 续照射 $15 \mathrm{~s}$ 后，体系达到了稳定的状态，此时偶氮苯 trans-和 cis-构型转变达到平衡. 图 $3 \mathrm{~b}$ 为该溶液在 530

(1)<smiles>N#CCN(CCN)c1ccccc1-c1ccc([N+](=O)[O-])cc1</smiles><smiles>NCCN(CCN)c1ccc(N=C=Nc2ccccc2)cc1</smiles>

(2)<smiles>C#CCOCCOCCOCCOCCOCC#CC(=O)OC(=O)C(C)(C)Br</smiles>

(3)<smiles>C#CCOCCCOCCOCCCOCCOCC#CC(C)(C)COCCOCCOCCOCCOCc1cn(CCN(CCn2cc(C)nn2)c2ccc(N=Cc3ccccc3)cc2)nn1</smiles>

图式 1 交替共聚物 $\mathrm{P}\left(\mathrm{EG}_{4}-a-\mathrm{NAzo}\right)$ 的合成步骤

Scheme 1 Synthesis steps of alternating copolymer $\mathrm{P}_{\left(\mathrm{EG}_{4}-a-\mathrm{NAzo}\right)}$ 
(a)

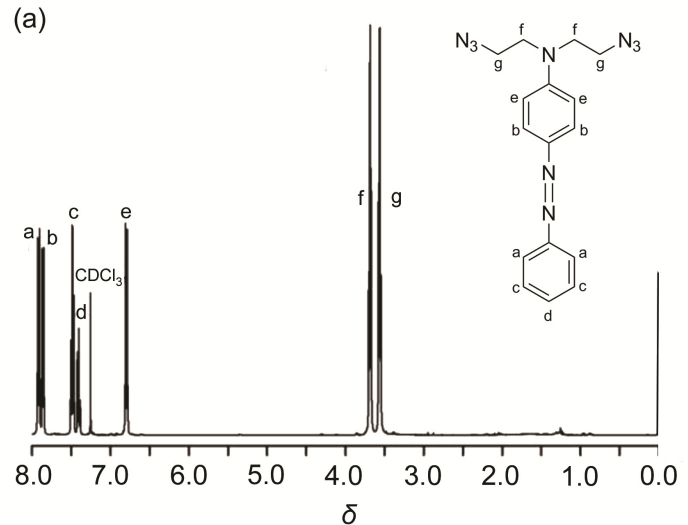

(c)
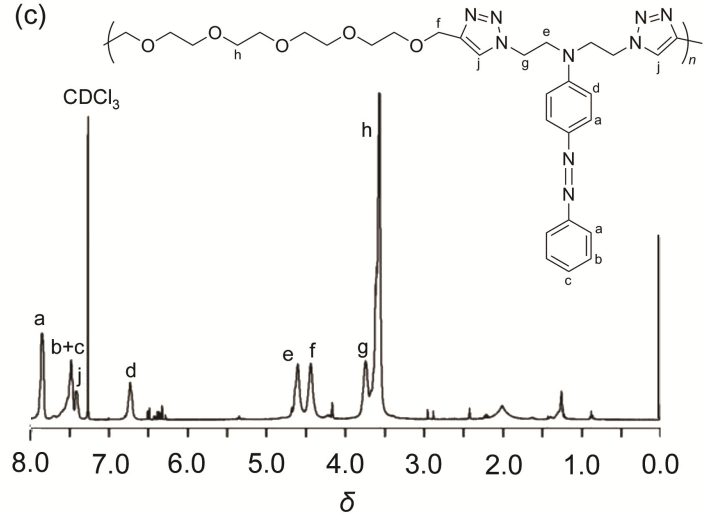

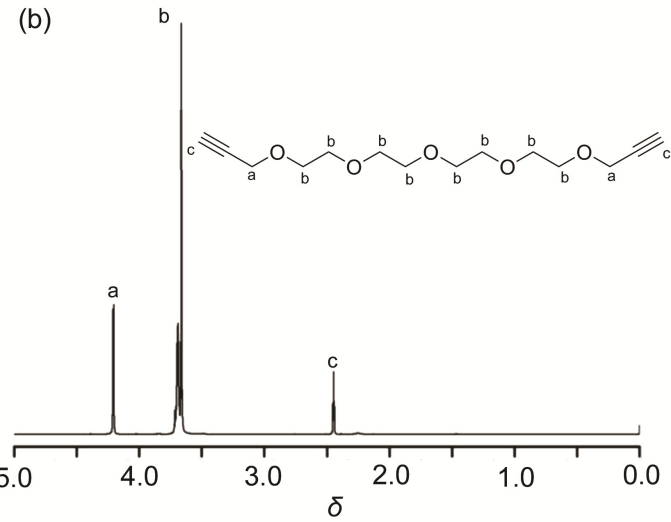

(d)

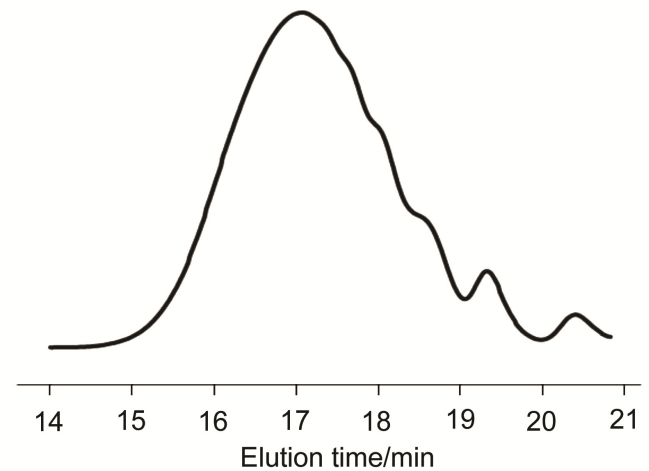

图 1 (a) $\mathrm{NAzo}_{-} \mathrm{N}_{3}$, (b) $\mathrm{PAEG}_{4}$ 和(c) $\mathrm{P}\left(\mathrm{EG}_{4}-a-\mathrm{NAzo}\right)$ 的 ${ }^{1} \mathrm{H}$ NMR 谱图及(d) $\mathrm{P}\left(\mathrm{EG}_{4}-a-\mathrm{NAzo}\right)$ 的 $\mathrm{GPC}$ 曲线

Figure $1{ }^{1} \mathrm{H}$ NMR spectra of $\mathrm{NAzo}^{-\mathrm{N}_{3}}(\mathrm{a}), \mathrm{PAEG}_{4}$ (b), $\mathrm{P}\left(\mathrm{EG}_{4}-a-\mathrm{NAzo}\right)(\mathrm{c})$ and GPC spectrum of $\mathrm{P}\left(\mathrm{EG}_{4}-a-\mathrm{NAzo}\right)(\mathrm{d})$
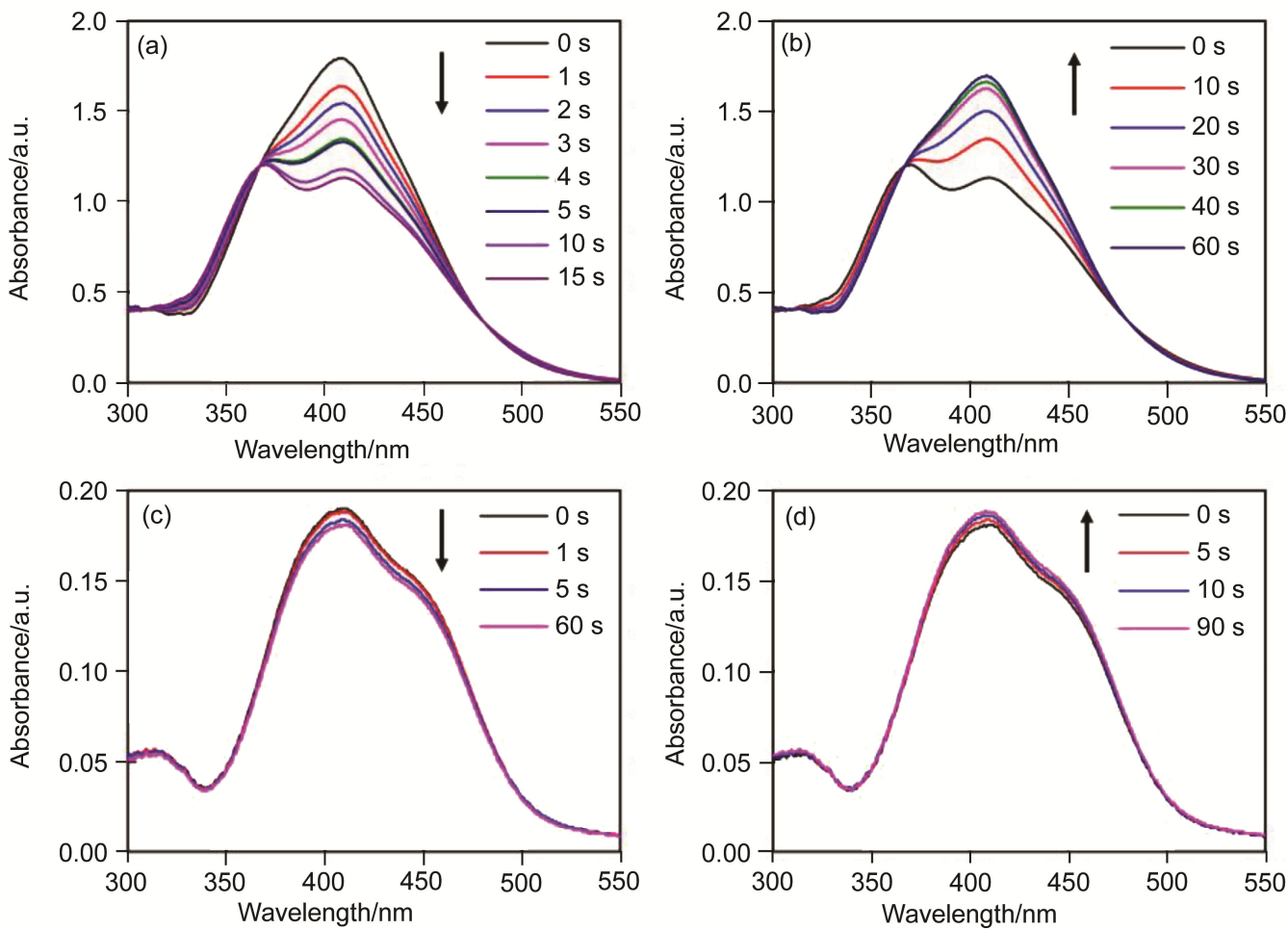

图 $2 \mathrm{P}\left(\mathrm{EG}_{4}-a-\mathrm{NAzo}\right)$ 聚合物 $\mathrm{DMF}$ 溶液在(a) 450 和(b) $530 \mathrm{~nm}$ 光照下，以及胶束水溶液在(c) 450 和(d) $530 \mathrm{~nm}$ 光照下的紫外吸收 光谱图

Figure 2 UV-Vis absorption spectra of $\mathrm{P}\left(\mathrm{EG}_{4}-a-\mathrm{NAzo}\right)$ in DMF upon irradiation of (a) 450 and (b) $530 \mathrm{~nm}$ light, and $\mathrm{P}\left(\mathrm{EG}_{4}-a-\mathrm{NAzo}\right)$ micelles in water upon irradiation of (c) 450 and (d) $530 \mathrm{~nm}$ light 
$\mathrm{nm}$ 绿光照射下的回复过程的 UV-Vis 谱图, 经过 $60 \mathrm{~s}$ 照 射后, cis-构型重新回复为原先较为稳定的 trans-构型. $\mathrm{P}\left(\mathrm{EG}_{4}-a-\mathrm{NAzo}\right)$ 在 $450 \mathrm{~nm}$ 蓝光和 $530 \mathrm{~nm}$ 绿光下的光致 异构化构型转变可以重复至少 5 次以上, 吸收峰能够完 全回复到最初的强度, 这表明该聚合物光致异构化是稳 定且可逆的. 使用 $450 \mathrm{~nm}$ 的蓝光照射 $\mathrm{P}\left(\mathrm{EG}_{4}-a\right.$-NAzo) 胶 束溶液, 与 DMF 溶液相比, 在相同光强相同照射时间 下, 胶束溶液纵向的吸光度变化明显减小, 这是因为在 胶束溶液中, 疏水部分聚集在一起, 导致偶氮苯基团的 运动能力下降, 异构化速率变慢, 在 $450 \mathrm{~nm}$ 蓝光照射 $60 \mathrm{~s}$ 以后达到平衡稳定的状态 (图 2c). 通过 $530 \mathrm{~nm}$ 绿光 照射, 也可以将顺式偶氮苯恢复为较为稳定的反式结 构, 照射 $90 \mathrm{~s}$ 后, cis-构型重新变为原先的 trans-构型, 这一转变同样是完全可逆的(图 2d). 此外, $\mathrm{P}_{\left(\mathrm{EG}_{4}-a-\right.}$ NAzo)独特的交替两亲性拓扑结构使得胶束中的偶氮苯 基团难以进行规则的 $J$-型和 $H$-型聚集 ${ }^{[15]}$, 因此胶束溶 液的紫外吸收峰并没有发生红移或是蓝移.

\section{3 交替共聚物 $P\left(E_{-}-a-N A z o\right)$ 的溶液自组装行为}

按照实验部分中的胶束制备步骤, 将 $\mathrm{P}\left(\mathrm{EG}_{4}-a-\right.$ $\mathrm{NAzo}$ )分别配制成浓度为 0.1 和 $0.05 \mathrm{mg} \cdot \mathrm{mL}^{-1}$ 的聚合物 胶束水溶液, 使用透射电镜(TEM)对其形貌进行观察表 征, 结果如图 3 所示.

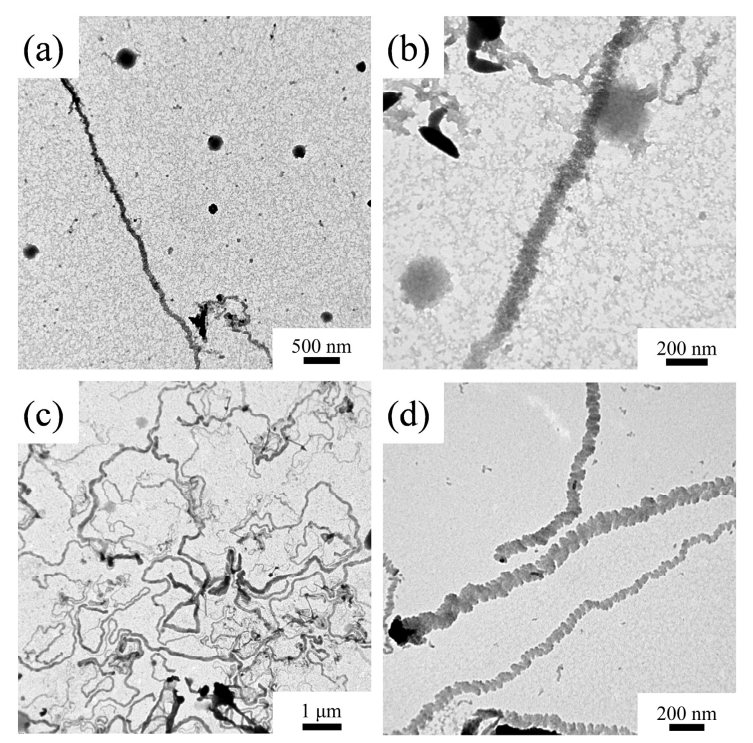

图 3 (a, b) 0.1 和 $(\mathrm{c}, \mathrm{d}) 0.05 \mathrm{mg} \bullet \mathrm{mL}^{-1}$ 共聚物 $\mathrm{P}\left(\mathrm{EG}_{4}-a-\mathrm{NAzo}\right)$ 胶束水溶液的 TEM 图

Figure 3 TEM of alternating $\mathrm{P}\left(\mathrm{EG}_{4}-a\right.$-NAzo $)$ with concentrations of $0.1(\mathrm{a}, \mathrm{b})$ and $0.05 \mathrm{mg} \cdot \mathrm{mL}^{-1}(\mathrm{c}, \mathrm{d})$ in aqueous solution

浓度 $0.1 \mathrm{mg} \cdot \mathrm{mL}^{-1}$ 的聚合物胶束形貌如图 $3 \mathrm{a}$ 和 $3 \mathrm{~b}$ 所示, 组装体以蠕虫状结构为主, 其长度为 $5 \mu \mathrm{m}$ 以上, 宽度为 $70 \mathrm{~nm}$ 左右，同时也存在 $200 \sim 300 \mathrm{~nm}$ 直径的球 状大复合胶束. 浓度 $0.05 \mathrm{mg} \cdot \mathrm{mL}^{-1}$ 的聚合物胶束形貌如
图 3c 和 $3 \mathrm{~d}$ 所示, 组装体均为蠕虫状, 其长度可达 10 $\mu \mathrm{m}$, 宽度为 $110 \mathrm{~nm}$. 对蠕虫状组装体进一步观察(图 3b 和 3d) 可见, 其内部存在堆积的二级结构. 根据经典大 分子自组装理论 ${ }^{[1 b]}$, 亲水部分体积分数较高时首先得 到球状胶束，随着疏水部分体积分数增加，得到棒状胶 束等一系列形态，当疏水部分体积分数非常高时才会得

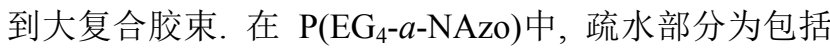
三唑环在内的 NAzo 链段, 亲水部分为 $\mathrm{EG}_{4}$ 链段, 疏水 部分的分子量大, 加之芳环结构刚性较强, 使得疏水部 分体积分数很大; 而亲水部分分子量小，链段柔软，体 积分数小，应倾向于形成大复合胶束. 但是，特殊的交 替刚-柔型疏水/亲水拓扑结构也使得疏水部分难以很好 地团聚在一起, 更难以形成在普通偶氮苯聚合物组装体 中常见的 $J$-或 $H$-堆叠, 这也被紫外光谱研究所证实. 因 此我们推测, $\mathrm{P}\left(\mathrm{EG}_{4}-a\right.$-NAzo $)$ 的自组装可能并不完全遵 循大分子自组装的规律: 在浓度较高时, 大复合胶束得 以形成, 并能稳定存在一段时间, 所以可观察到大复合 胶束与蠕虫状组装体共存; 而在浓度较低时, 首先形成 的大复合胶束并不稳定，会进一步解体形成类似蠕虫状 的组装体，使整个体系的熵增加. 这些蠕虫胶束的节则 是由团聚的疏水段 NAzo 构成的，由于一条分子链上有 多个疏水段，因此一部分 $\mathrm{P}\left(\mathrm{EG}_{4}-a-\mathrm{NAzo}\right)$ 的疏水段通过 在不同的节内团聚使得各个部分得以相连，从而形成具 有多级结构的类蠕虫状胶束. 这一设想还有待于进一步 实验研究证实.

\section{2 结论}

通过叠氮一炔基的点击反应，制备了一种新型的含 偶氮苯侧链两亲性交替共聚物 $\mathrm{P}\left(\mathrm{EG}_{4}-a-\mathrm{NAzo}\right) . \mathrm{P}\left(\mathrm{EG}_{4}-a\right.$ $\mathrm{NAzo}$ )在 DMF 溶液和胶束溶液中的光致异构化行为表 明，偶氮苯基团在胶束中不能形成有序的堆叠. 该交替 共聚物可以在溶液中组装成多级结构的蠕虫状聚集体, 并且在较低浓度下更有利于蠕虫状聚集体的形成. 这些 异于普通侧链偶氮苯嵌段聚合物的行为均与其特殊的 交替亲-疏水刚/柔拓扑结构有关. $\mathrm{P}\left(\mathrm{EG}_{4}-a-\mathrm{NAzo}\right)$ 的成功 合成, 丰富了含偶氮苯单元聚合物的结构与类型, 也为 功能性交替共聚物的研究和制备提供了新的策略.

\section{3 实验部分}

\section{1 仪器与试剂}

$N, N$-二羟乙基苯胺 ( $N$-phenyldiethanolamine, $98 \%$, Adamas)，吡啶(pyridine，99\%，Adamas)，对甲苯磺酰氯 ( $\mathrm{TsCl}, 99 \%$, Adamas), 苯胺(aniline, 98\%, Adamas), 四氟 喼酸水溶液(fluoroboric acid, 40\%, Adamas), 叔丁醇钾 (potassium tert-butoxide, 99\%, Adamas)，三缩四乙二醇 
(bis[2-(2-hydroxyethoxy)ethyl] ether, 99\%, Adamas), 溴 化亚铜(copper(I) bromide, 99\%, Adamas), 五甲基二乙烯 三胺(PMDETA, 99\%, Adamas), 四氢呋喃(THF, AR, 上 海泰坦), $N, N$-二甲基甲酰胺( $\mathrm{DMF}, \mathrm{AR}$, 上海泰坦). 石 油醚(PE, 精馏级, 上海泰坦), 乙酸乙酯(EA, AR, 上海 泰坦). 实验用水为超纯水, 自制.

场发射扫描电子显微镜(FESEM)(日本日立公司, S-4800 型); 透射电子显微镜 (TEM) (JEOL 公司, JEM-1400 型); Bruker AVANCE III-400MHz 核磁共振 谱仪 (内标试剂采用 $\mathrm{CDCl}_{3}$ ); 光致异构化: 采用紫外 LED 灯, 选取特定波长, 对聚合物溶液以及胶束溶液进 行照射(Uvata 公司, UP114 型).

\section{2 实验方法}

3.2.1 $\mathrm{TsCl}$ 取代生成 $N, N$-二甲苯磺酰乙基苯胺 (NPDAOTs)

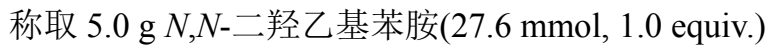
置于 $250 \mathrm{~mL}$ 三颈烧瓶中, 加入 $60 \mathrm{~mL}$ 吡啶, 在冰水浴条 件下辅以适当搅拌溶解上述单体. 然后称取 $12.7 \mathrm{~g}$ 对甲 苯磺酰氯( $66.3 \mathrm{mmol}, 2.4$ equiv.), 并缓慢将其加入到上 述溶液中, 始终保持冰水浴氛围并且辅以适当搅拌. 随 着反应进行, 整个体系逐渐变得浑浊. 反应进行了 $3 \mathrm{~h}$ 后, 停止反应. 通过旋转蒸发将吡啶除去溶剂除去, 然 后在强烈搅拌下向混合体系中加入大量冰水, 可以析出 白色固体. 再经过抽滤除去滤液得到白色固体, 使用冰 甲醇、冰乙醚对该白色固体进行多次洗涤，除去多余的 对甲苯磺酰氯, 真空干燥后得到 $8.5 \mathrm{~g}$ 白色固体 NPDAOTS, 产率 63\%. ${ }^{1} \mathrm{H}$ NMR $\left(400 \mathrm{MHz}, \mathrm{CDCl}_{3}\right) \delta$ : $7.71(\mathrm{~d}, J=8.3 \mathrm{~Hz}, 4 \mathrm{H}), 7.27$ (d, $J=8.7 \mathrm{~Hz}, 4 \mathrm{H}), 7.13$ (dd, $J=8.7,7.4 \mathrm{~Hz}, 2 \mathrm{H}), 6.70$ (t, $J=7.3 \mathrm{~Hz}, 1 \mathrm{H}), 6.43$ (d, $J=$ $8.2 \mathrm{~Hz}, 2 \mathrm{H}), 4.08(\mathrm{t}, J=6.1 \mathrm{~Hz}, 4 \mathrm{H}), 3.55(\mathrm{t}, J=6.1 \mathrm{~Hz}$, $4 \mathrm{H}), 2.42$ (s, 6H).

3.2.2 叠氮取代生成 $N, N-$ 二叠氮乙基苯胺 $\left(\mathrm{NPDA}-\mathrm{N}_{3}\right)$

室温下, 称取 $1.5 \mathrm{~g}$ NPDAOTS 固体(3.07 mmol, 1.0 equiv.)置于 $250 \mathrm{~mL}$ 三颈烧瓶中, 使用移液枪吸取 $10 \mathrm{~mL}$ DMF 溶剂, 加入到烧瓶中以溶解上述单体, 称取 $2.0 \mathrm{~g}$ $\mathrm{NaN}_{3}$ 固体(30.77 mmol, 10.0 equiv.), 通过滤纸漏斗缓慢 小心加入烧瓶. 往三颈烧瓶中通入氮气, 升温至 $80{ }^{\circ} \mathrm{C}$, 适当搅拌, 反应 $12 \mathrm{~h}$, 始终保持在氮气氛围下. 随后, 停止加热并冷却至室温. 通过自然过滤除去过量的叠氮 化钠, 得到黄色油状液体, 加入适量的去离子水溶解过 量的叠氮化钠, 随后使用 $\mathrm{CH}_{2} \mathrm{Cl}_{2}$ 萃取数次, 经无水 $\mathrm{Na}_{2} \mathrm{SO}_{4}$ 干燥，旋蒸得到 $0.6 \mathrm{~g}$ 黄色油状液体，产率 84\%. ${ }^{1} \mathrm{H}$ NMR (400 MHz, CDCl3) $\delta: 7.29 \sim 7.23$ (m, 2H), 6.78 (t, $J=7.3 \mathrm{~Hz}, 1 \mathrm{H}), 6.73(\mathrm{~d}, J=8.0 \mathrm{~Hz}, 2 \mathrm{H}), 3.59$ (t, $J=6.1$
$\mathrm{Hz}, 4 \mathrm{H}), 3.47$ (t, $J=6.1 \mathrm{~Hz}, 4 \mathrm{H})$.

\subsection{3 重氮盐的合成}

称取 $4.0 \mathrm{~g}$ 苯胺 ( $42.95 \mathrm{mmol}, 1.0$ equiv.)放置于 250 $\mathrm{mL}$ 三颈烧瓶中，随后往烧瓶里加入质量分数为 $40 \%$ 的 $\mathrm{HBF}_{4}$ 水溶液 (13.5 mL)中, 苯胺在 $\mathrm{HBF}_{4}$ 为白色沉淀, 无 法溶解形成澄清透明的溶液, 将 $3.3 \mathrm{~g}$ 亚硫酸钠(47.25 mmol, 1.1 equiv.)溶解于冰水溶液后再逐滴缓慢加入三 颈烧瓶中, 白色沉淀溶解, 溶液变得澄清, 反应混合物 在冰水浴环境中强烈搅拌, 出现乳白色沉淀, 反应 45 min 后, 将混合物抽滤，使用冰乙醚洗涤沉淀多次，得 到 $5.8 \mathrm{~g}$ 白色固体, 产率 70\%, 直接用于下一步反应.

取 $1.2 \mathrm{~g}$ 所得白色固体(6.46 mmol, 1.0 equiv.)以及 $1.5 \mathrm{~g} \mathrm{NPDA}^{-\mathrm{N}_{3}}$ 固体 $(6.46 \mathrm{mmol}, 1.0$ equiv.) 放置于三颈 烧瓶中，使用移液枪往烧瓶里加入 $10 \mathrm{~mL}$ DMF 以溶解 上述固体, 全程使用冰水浴作为反应环境, 并在适当摚 拌下进行反应, 反应过夜. 用水和 $\mathrm{CH}_{2} \mathrm{Cl}_{2}$ 萃取反应混合 物, 经由饱和 $\mathrm{NaCl}$ 水溶液洗涤, 无水 $\mathrm{Na}_{2} \mathrm{SO}_{4}$ 干燥后, 旋蒸, 色谱层析柱分离(展开剂: 石油醚/乙酸乙酯, $V$ : $V=7: 1$ ), 得到 $0.9 \mathrm{~g}$ 橙色固体, 产率 $40 \% .{ }^{1} \mathrm{H}$ NMR $\left(400 \mathrm{MHz}, \mathrm{CDCl}_{3}\right) \delta: 7.92(\mathrm{~d}, \quad J=9.1 \mathrm{~Hz}, 2 \mathrm{H}), 7.88 \sim$ $7.83(\mathrm{~m}, 2 \mathrm{H}), 7.49$ (dd, $J=10.3,4.7 \mathrm{~Hz}, 2 \mathrm{H}), 7.43 \sim 7.36$ $(\mathrm{m}, 1 \mathrm{H}), 6.80(\mathrm{~d}, J=9.1 \mathrm{~Hz}, 2 \mathrm{H}), 3.69(\mathrm{t}, J=6.0 \mathrm{~Hz}, 4 \mathrm{H})$, $3.56(\mathrm{t}, J=6.0 \mathrm{~Hz}, 4 \mathrm{H})$.

\subsection{4 端丙炔基三缩四乙二醇 $\left(\mathrm{PAEG}_{4}\right)$ 的合成}

称取 $4.9 \mathrm{~g}$ 叔丁醇钾 $(44.0 \mathrm{mmol}, 2.2$ equiv.) 放置于三 颈烧瓶中, 使用量筒称量 $100 \mathrm{~mL}$ THF 加入到烧瓶中, 在冰水浴条件下溶解叔丁醇钾, 并且持续通入氮气. 称 取 $3.9 \mathrm{~g}$ 三缩四乙二醇 $(20.0 \mathrm{mmol}, 1.0$ equiv.)通过注射器 缓慢加入并剧烈搅拌. 反应 $0.5 \mathrm{~h}$ 后, 称取 $5.2 \mathrm{~g} \mathrm{3}$-溴丙 炔(44.0 mmol, 2.2 equiv.)通过注射器加入三颈烧瓶, 在 冰水浴条件下反应 $3 \mathrm{~h}$. 粗产物通过色谱层析柱分离(展 开剂: 石油梄/乙酸乙酯, $V: V=3: 2)$, 得到 $3.6 \mathrm{~g}$ 淡黄 色油状液体, 产率 $66 \% .{ }^{1} \mathrm{H}$ NMR $\left(400 \mathrm{MHz}, \mathrm{CDCl}_{3}\right) \delta$ : $4.21(\mathrm{~d}, J=2.4 \mathrm{~Hz}, 4 \mathrm{H}), 3.74 \sim 3.62(\mathrm{~m}, 16 \mathrm{H}), 2.45(\mathrm{t}, J=$ $2.4 \mathrm{~Hz}, 2 \mathrm{H})$

3.2.5 聚(三缩四乙二醇一偶氮苯二乙胺) $\left[\mathrm{P}\left(\mathrm{EG}_{4}-a\right.\right.$ NAzo)]的合成

称取 $3.4 \mathrm{mg}$ 澳化亚铜 $(0.024 \mathrm{mmol}, 0.05$ equiv.)置于 施兰克管中, 封上橡胶塞并持续通入氮气. 然后, 称取 $177.0 \mathrm{mg}$ 的 NAzo-N $\mathrm{N}_{3}(0.528 \mathrm{mmol}, 1.1$ equiv.)和 130.0 $\mathrm{mg}$ 的 $\mathrm{PAEG}_{4}(0.48 \mathrm{mmol}, 1.0$ equiv.), 分别溶解在 $1 \mathrm{~mL}$ DMF 中, 使用针筒加入到反应管中. 采用液氮冷冻-抽 真空-通氮气的操作来确保体系中无水无氧的环境, 重 复 3 次, 第 2 次之后, 称取 $8.5 \mu \mathrm{L}$ 五甲基二乙烯三胺 (0.048 mmol, 0.1 equiv.)溶解于 $1 \mathrm{~mL}$ DMF 后使用微量进 
样器注射到反应管中, 重复最后 1 次冻抽、通氮气操作, 将反应管置于 $35{ }^{\circ} \mathrm{C}$ 油浴中反应 $24 \mathrm{~h}$. 停止反应后, 采 用 60 目中性氧化铝除去 $\mathrm{Cu}(\mathrm{I})$ 催化剂, 浓缩溶液后在大 量冰乙醚中沉淀, 再用 DMF 溶解再沉淀, 循环 3 次. 最 后得到 $156.0 \mathrm{mg}$ 橙色固体, 产率 54\%. ${ }^{1} \mathrm{H}$ NMR (400 $\left.\mathrm{MHz}, \mathrm{CDCl}_{3}\right) \delta: 7.98 \sim 7.85(\mathrm{~m}, \mathrm{ArH}), 7.51 \sim 7.45(\mathrm{~m}, \mathrm{ArH}$ on triazole), $7.45 \sim 7.38(\mathrm{~m}, \mathrm{ArH}), 6.80 \sim 6.72(\mathrm{~m}, \mathrm{ArH})$, $4.69 \sim 4.62\left(\mathrm{~m}, \mathrm{CH}_{2} \mathrm{~N}\right), 4.51 \sim 4.40\left(\mathrm{~m}, \mathrm{O}-\mathrm{CH}_{2}\right.$-triazole $)$, $3.82 \sim 3.74\left(\mathrm{~m}, \mathrm{CH}_{2} \mathrm{CH}_{2}\right.$-triazole), $3.70 \sim 3.50\left(\mathrm{~m}, \mathrm{OCH}_{2}\right)$.

\subsection{6 胶束溶液的制备过程}

首先, 将合成的含偶氮苯侧链两亲性交替共聚物 $\mathrm{P}\left(\mathrm{EG}_{4}-a-\mathrm{NAzo}\right)$ 溶解在良溶剂 DMF 中, 配置成不同浓度 的溶液, 随后在恒温震荡培养箱中放置 $24 \mathrm{~h}$, 促使其充 分溶解. 然后取 $1 \mathrm{~mL}$ 聚合物溶液, 在室温下使用微量 注射洜以流速 $1 \mathrm{~mL} \cdot \mathrm{h}^{-1}$ 往其中加入 $2 \mathrm{~mL}$ 去离子水并低 速搅拌, 待水滴加完毕后, 再搅拌 $2 \mathrm{~h}$, 让聚合物链段在 混合溶剂中充分组装, 随后立即加入 $10 \mathrm{~mL}$ 去离子水来 进行形貌的固定, 稳定 $0.5 \mathrm{~h}$ 后, 使用移液枪把胶束溶液 转移到透析袋中(截留分子量为 3500), 透析过程持续 3 $\mathrm{d}$, 一天更换 2 3 次去离子水, 以除去有机溶剂.

辅助材料(Supporting Information) 中间体 NPDAOTs 和 NPDA-N 的的 $^{1} \mathrm{H}$ NMR 图谱. 这些材料可以免费从 本刊网站(http://sioc-journal.cn/)上下载.

\section{References}

[1] (a) Thorkelsson, K.; Bai, P.; Xu, T. Nano Today 2015, 10, 48. (b) Mai, Y.; Eisenberg, A. Chem. Soc. Rev. 2012, 41, 18.

[2] (a) Howe, D. H.; Hart, J. L.; McDaniel, R. M.; Taheri, M. L.; Magenau, A. J. ACS Macro Lett. 2018, 7, 1503.

(b) Zhang, L.; Eisenberg, A. Science 1995, 268, 1728.

(c) Shen, H.; Eisenberg, A. Angew. Chem., Int. Ed. 2000, 39, 3310

(d) Zhang, L.; Eisenberg, A. J. Am. Chem. Soc. 1996, 118, 3168.

[3] (a) Gao, F.; Xing, Y.; Yao, Y.; Sun, L.; Sun, Y.; He, X.; Lin, S. Polym. Chem. 2017, 8, 7529. (b) Sun, L.; Gao, F.; Shen, D.; Liu, Z.; Yao, Y.; Lin, S. Polym. Chem. 2018, 9, 2977.

[4] (a) Dong, R.; Zhu, B.; Zhou, Y.; Yan, D.; Zhu, X. Angew. Chem., Int. Ed. 2012, 51, 11633 .

(b) Ren, H.; Chen, D.; Shi, Y.; Yu, H.; Fu, Z. Polym. Chem. 2015, 6, 270 .

(c) Ren, H.; Chen, D.; Shi, Y.; Yu, H.; Fu, Z. Polymer 2016, 97, 533.

[5] Yu, S.; Azzam, T.; Rouiller, I.; Eisenberg, A. J. Am. Chem. Soc. 2009, 131, 10557.

[6] Lv, J. A.; Liu, Y.; Wei, J.; Chen, E.; Qin, L.; Yu, Y. Nature 2016, 537,179 .

[7] (a) Takeshima, T.; Liao, W. Y.; Nagashima, Y.; Beppu, K.; Hara, M.; Nagano, S.; Seki, T. Macromolecules 2015, 48, 6378.

(b) Gao, F.; Wang, W.; Li, X.; Li, L.; Lin, J.; Lin, S. J. Colloid Interface Sci. 2016, 468, 70.

(c) Kong, X.; Wang, X.; Luo, T.; Yao, Y.; Li, L.; Lin, S. ACS Appl. Mater. Interfaces 2017, 9, 19345.

(d) Lee, S.; Kang, H. S.; Park, J. K. Adv. Mater. 2012, 24, 2069.

(e) Wang, W.; Shen, D.; Li, X.; Yao, Y.; Lin, J.; Wang, A.; Yu, J.; Wang, Z. L.; Hong, S. W.; Lin, Z; Lin, S. Angew. Chem., Int. Ed. 2018, 130, 2161.

(f) Wang, W.; Du, C.; Wang, X.; He, X.; Lin, J.; Li, L.; Lin, S. Angew. Chem., Int. Ed. 2014, 126, 12312. (g) Fu, S.; Zhao, Y. Macromolecules 2015, 48, 5088.

[8] Moses, J. E.; Moorhouse, A. D. Chem. Soc. Rev. 2007, 36, 1249.

[9] Chen, J.; Yu, C.; Shi, Z.; Yu, S.; Lu, Z.; Jiang, W.; Zhang, M.; He, W.; Zhou, Y.; Yan, D. Angew. Chem., Int. Ed. 2015, 54, 3621

[10] Xu, Q.; Huang, T.; Li, S.; Li, K.; Li, C.; Liu, Y.; Wang, Y.; Yu, C.; Zhou, Y. Angew. Chem., Int. Ed. 2018, 57, 8043.

[11] (a) Lee, L. V.; Mitchell, M. L.; Huang, S.-J.; Fokin, V. V.; Sharpless, K. B.; Wong, C.-H. J. Am. Chem. Soc. 2003, 125, 9588. (b) Li, Z. a.; Yu, G.; Hu, P.; Ye, C.; Liu, Y.; Qin, J.; Li, Z. Macromolecules 2009, 42, 1589.

[12] (a) Hii, K. K.; Thornton-Pett, M.; Jutand, A.; Tooze, R. P. Organometallics 1999, 18, 1887.

(b) Lee, S. J.; Lee, S. S.; Lee, J. Y.; Jung, J. H. Chem. Mater. 2006 $18,4713$.

[13] Wu, S.; Yu, X.; Huang, J.; Shen, J.; Yan, Q.; Wang, X.; Wu, W.; Luo, Y.; Wang, K.; Zhang, Q. J. Mater. Chem. 2008, 18, 3223.

[14] (a) Weis, P.; Wu, S. Macromol. Rapid Commun. 2018, 39, 1700220. (b) Weis, P.; Tian, W.; Wu, S. Chem.-Eur. J. 2018, 24, 6494.

[15] (a) Wu, S.; Wang, L.; Kroeger A.; Wu, Y.; Zhang, Q.; Bubeck, C. Soft Mater. 2011, 7, 11535.

(b) Li, Y.; Deng, Y.; Tong, X.; Wang, X. Macromolecules 2006, 39, 1108 . 